\title{
Application to Coupled Fixed-Point Theorems on Complex Partial b-Metric Space
}

\author{
Gunaseelan Mani, ${ }^{1}$ Lakshmi Narayan Mishra ${ }^{(D)}{ }^{2}$ Vishnu Narayan Mishra $\left(\mathbb{D},{ }^{3}\right.$ \\ Imran Abbas Baloch $\left(\mathbb{0},{ }^{4,5}\right.$ and Manuel De La Sen $\mathbb{1}^{6}$ \\ ${ }^{1}$ Department of Mathematics, Sri Sankara Arts and Science College (Autonomous), Affiliated to Madras University, \\ Enathur, Kanchipuram, Tamil Nadu, 631 561, India \\ ${ }^{2}$ Department of Mathematics, School of Advanced Sciences, Vellore Institute of Technology (VIT) University, Vellore 632014, \\ Tamil Nadu, India \\ ${ }^{3}$ Department of Mathematics, Indira Gandhi National Tribal University, Laipur, Amarkantak, Anuppur, Madhya Pradesh, \\ 484887, India \\ ${ }^{4}$ Abdus Salam School of Mathematical Sciences GC University, Lahore, Pakistan \\ ${ }^{5}$ Higher Education Department Govt. College for Boys Gulberg, Lahore, Punjab, Pakistan \\ ${ }^{6}$ Institute of Research and Development of Processors University of the Basque Country Campus of Leioa (Bizkaia), \\ Leioa 48940, Spain
}

Correspondence should be addressed to Imran Abbas Baloch; iabbasbaloch@gmail.com

Received 27 August 2020; Revised 8 September 2020; Accepted 13 September 2020; Published 26 November 2020

Academic Editor: Jen-Chih Yao

Copyright (C) 2020 Gunaseelan Mani et al. This is an open access article distributed under the Creative Commons Attribution License, which permits unrestricted use, distribution, and reproduction in any medium, provided the original work is properly cited.

In this paper, we prove coupled fixed-point theorems in complex partial b-metric space. The proved results generalize and extend some of the well-known results in the literature. We also give some applications of our main results.

\section{Introduction}

The notion of b-metric space was introduced by Backhtin [1] in 1989, and Czerwik [2] extended the results of metric spaces. The notion of complex valued metric spaces was introduced by Azam et al. [3] in 2011 who also proved some common fixed-point theorems under the contraction condition. In 2013, Rao et al. [4] introduced the concept of complex valued b-metric space which is more general than the well-known complex valued metric space and also proved common fixed-point theorem under the contraction condition. In 2017, Dhivya and Marudai [5] introduced the notion of complex partial metric space and also proved common fixed-point theorems under the contraction condition of rational expression. In 2019, Gunaseelan [6] introduced the notion of complex partial b-metric space and also proved fixed-point theorem under the contractive condition. Some interesting concepts and applications have been studied by many authors, and important results have been obtained in [7-12]. In this paper, we prove coupled fixed-point theorems in complex partial b-metric space.

In the next section, we give basic definitions, examples, and primary results for the better understanding of our major results presented in this research paper.

\section{Preliminaries}

Let $\mathbb{C}$ be the set of complex numbers and $\eta_{1}, \eta_{2}, \eta_{3} \in \mathbb{C}$. Define a partial order $\preceq$ on $\mathbb{C}$ as follows: $\eta_{1} \preceq \eta_{2}$ if and only if $\operatorname{Re}\left(\eta_{1}\right) \leq \operatorname{Re}\left(\eta_{2}\right), \operatorname{Im}\left(\eta_{1}\right) \leq \operatorname{Im}\left(\eta_{2}\right)$.

Consequently, one can infer that $\eta_{1} \preceq \eta_{2}$ if one of the following conditions is satisfied:

(i) $\operatorname{Re}\left(\eta_{1}\right)=\operatorname{Re}\left(\eta_{2}\right), \operatorname{Im}\left(\eta_{1}\right)<\operatorname{Im}\left(\eta_{2}\right)$

(ii) $\operatorname{Re}\left(\eta_{1}\right)<\operatorname{Re}\left(\eta_{2}\right), \operatorname{Im}\left(\eta_{1}\right)=\operatorname{Im}\left(\eta_{2}\right)$

(iii) $\operatorname{Re}\left(\eta_{1}\right)<\operatorname{Re}\left(\eta_{2}\right), \operatorname{Im}\left(\eta_{1}\right)<\operatorname{Im}\left(\eta_{2}\right)$ 
(iv) $\operatorname{Re}\left(\eta_{1}\right)=\operatorname{Re}\left(\eta_{2}\right), \operatorname{Im}\left(\eta_{1}\right)=\operatorname{Im}\left(\eta_{2}\right)$

In particular, we write $\eta_{1} \precsim \eta_{2}$ if $\eta_{1} \neq \eta_{2}$ and one of (i), (ii), and (iii) is satisfied and we write $\eta_{1} \prec \eta_{2}$ if only (iii) is satisfied. Notice that

(a) If $0 \prec \eta_{1} \precsim \eta_{2}$, then $\left|\eta_{1}\right|<\left|\eta_{2}\right|$

(b) If $\eta_{1} \prec \eta_{2}$ and $\eta_{2} \prec \eta_{3}$, then $\eta_{1} \prec \eta_{3}$

(c) If $\lambda, \beta \in \mathbb{R}$, and $\lambda \leq \beta$, then $\lambda a_{1} \prec \beta a_{1}$ for all $0 \prec a_{1} \in \mathbb{C}$

Definition 1 (see [4]). Let $Y$ be a nonvoid set and let $s \geq 1$ be a given real number. A function $d: Y \times Y \longrightarrow \mathbb{C}$ is called a complex valued b-metric on $Y$ if for all $a, b, c \in Y$ the following conditions are satisfied:

(i) $0 \prec d(a, b)$ and $d(a, b)=0$ if and only if $a=b$

(ii) $d(a, b)=d(b, a)$

(iii) $d(a, b) \prec s[d(a, c)+d(c, b)]$

The pair $(Y, d)$ is called a complex valued $b$-metric space.

Here, $\mathbb{C}^{+}\left(=\left\{(a, b) \mid a, b \in \mathbb{R}^{+}\right\}\right)$and $\mathbb{R}^{+}(=\{a \in \mathbb{R} \mid a \geq 0\})$ denote the set of non-negative complex numbers and the set of non-negative real numbers, respectively. We now give the complex partial metric space.

Definition 2 (see [5]). A complex partial metric on a nonvoid set $\triangle$ is a function $\zeta_{c}: \triangle \times \triangle \longrightarrow \mathbb{C}^{+}$such that for all $e, f, g \in \triangle$ :

(i) $0 \prec \zeta_{c}(e, e) \prec \zeta_{c}(e, f)$ (small self - distances)

(ii) $\zeta_{c}(e, f)=\zeta_{c}(f, e)$ (symmetry)

(iii) $\zeta_{c}(e, e)=\zeta_{c}(e, f)=\zeta_{c}(f, f)$ if and only if $e=f$ (equality)

(iv) $\zeta_{c}(e, f) \prec \zeta_{c}(e, g)+\zeta_{c}(g, f)-\zeta_{c}(g, g)$ (triangularity)

A complex partial metric space is a pair $\left(\triangle, \zeta_{c}\right)$ such that $\triangle$ is a nonvoid set and $\zeta_{c}$ is the complex partial metric on $\triangle$.

Definition 3 (see [6]). A complex partial b-metric on a nonvoid set $\triangle$ is a function $\theta_{c b}: \triangle \times \triangle \longrightarrow \mathbb{C}^{+}$such that for all $e, f, g \in \triangle$ :

(i) $0 \prec \theta_{c b}(e, e) \prec \theta_{c b}(e, f)$ (small self - distances)

(ii) $\theta_{c b}(e, f)=\theta_{c b}(f, e)$ (symmetry)

(iii)

$\theta_{c b}(e, e)=\theta_{c b}(e, f)=\theta_{c b}(f, f) \Longleftrightarrow e=f$ (equality)

(iv) $\exists$ a real number $s \geq 1$ such that $\theta_{c b}(e, f) \prec s\left[\theta_{c b}(e\right.$, $\left.g)+\theta_{c b}(g, f)\right]-\theta_{c b}(g, g)$ (triangularity)

A complex partial b-metric space is a pair $\left(\triangle, \theta_{c b}\right)$ such that $\triangle$ is a nonvoid set and $\theta_{c b}$ is the complex partial b-metric on $\triangle$. The number $s$ is called the coefficient of $\left(\triangle, \theta_{c b}\right)$.

Remark 1 (see [6]). In a complex partial b-metric space $\left(\triangle, \theta_{c b}\right)$, if $e, f \in \triangle$ and $\theta_{c b}(e, f)=0$, then $e=f$, but the converse may not be true.
Remark 2 (see [6]). It is clear that every complex partial metric space is a complex partial b-metric space with coefficient $s=1$ and every complex valued b-metric is a complex partial b-metric space with the same coefficient and zero self-distance. However, the converse of this fact need not hold.

Example 1 (see [6]). Let $\triangle=\mathbb{R}^{+}$and $\theta_{c b}: \triangle \times \triangle \longrightarrow \mathbb{C}^{+}$be defined by $\theta_{c b}(e, f)=[\max \{e, f\}]^{3}+|e-f|^{3}+i\{[\max \{e$, $\left.f\}]^{3}+|e-f|^{3}\right\}, \quad \forall e, f \in \triangle$. Then, $\left(\triangle, \theta_{c b}\right)$ is a complex partial b-metric space with coefficient $s=2^{3}$, but it is neither a complex valued b-metric nor a complex partial metric.

Proposition 1 (see [6]). Let $\triangle$ be a nonvoid set such that $\zeta_{c}$ is a complex partial metric and $d$ is a complex valued $b$-metric with coefficient $s>1$ on $\triangle$. Then, the function $\theta_{c b}: \triangle \times \triangle \longrightarrow \mathbb{C}^{+}$defined by $\theta_{c b}(e, f)=\zeta_{c}(e, f)+d(e$, $f), \forall e, f \in \triangle$ is a complex partial b-metric on $\triangle$, that is, $\left(\triangle, \theta_{c b}\right)$ is a complex partial b-metric space.

Proposition 2 (see [6]). Let $\left(\triangle, \zeta_{c}\right)$ be a complex partial metric space, $r \geq 1$; then, $\left(\triangle, \theta_{c b}\right)$ is a complex partial $b$-metric space with coefficient $s=2^{r-1}$, where $\theta_{c b}$ is defined by $\theta_{c b}(e, f)=\left[\zeta_{c}(e, f)\right]^{r}$.

Every complex partial b-metric $\theta_{c b}$ on a nonvoid set $\triangle$ generates a topology $\tau_{c b}$ on $\triangle$ whose base is the family of open $\theta_{c b}$-balls $B_{\theta_{c b}}(e, \varepsilon)$ where $\tau_{c b}=\left\{B_{\theta_{c b}}(e, \varepsilon): e \in \triangle, \varepsilon>0\right\}$ and $B_{\theta_{c b}}(e, \varepsilon)=\left\{f \in \triangle: \theta_{c b}(e, f)<\varepsilon+\theta_{c b}(e, e)\right\}$. Now, we define Cauchy sequence and convergent sequence in complex partial b-metric spaces.

Definition 4 (see [6]). Let $\left(\triangle, \theta_{c b}\right)$ be a complex partial b-metric space with coefficient $s$. Let $\left\{e_{n}\right\}$ be any sequence in $\triangle$ and $e \in \triangle$. Then,

(i) The sequence $\left\{e_{n}\right\}$ is said to be convergent with respect to $\tau_{c b}$ and converges to $e$, if $\lim _{n \longrightarrow \infty} \theta_{c b}\left(e_{n}, e\right)=\theta_{c b}(e, e)$.

(ii) The sequence $\left\{e_{n}\right\}$ is said to be Cauchy sequence in $\left(\triangle, \theta_{c b}\right)$ if $\lim _{n, m \rightarrow \infty} \theta_{c b}\left(e_{n}, e_{m}\right)$ exists and is finite.

(iii) $\left(\triangle, \theta_{c b}\right)$ is said to be a complete complex partial b-metric space if for every Cauchy sequence $\left\{e_{n}\right\}$ in $\triangle$ there exists $e \in \triangle$ such that $\lim _{n, m \longrightarrow \infty} \theta_{c b}$ $\left(e_{n}, e_{m}\right)=\lim _{n \longrightarrow \infty} \theta_{c b}\left(e_{n}, e\right)=\theta_{c b}(e, e)$.

(iv) A mapping $T: \triangle \longrightarrow \triangle$ is said to be continuous at $e_{0} \in \triangle$ if for every $\varepsilon>0$, there exists $\delta>0$ such that $T\left(B_{\theta_{c b}}\left(e_{0}, \delta\right)\right) \subset B_{\delta_{c b}}\left(T\left(e_{0}, \varepsilon\right)\right)$.

Let $\triangle$ be a complex partial b-metric space and $B \subseteq \triangle$. A point $e \in \triangle$ is called an interior of set $B$, if there exists $0<r \in \mathbb{C}$ such that $B_{\theta_{c b}}(e, r)=\left\{f \in \triangle: \theta_{c b}(e, f)<\theta_{c b}(e\right.$, $e)+r\} \subseteq B$. A subset $B$ is called open, if each point of $B$ is an interior point of $B$. A point $e \in \triangle$ is said to be a limit point of $B$, for every $0<r \in \mathbb{C}, B_{\theta_{c b}}(e, r) \cap(B-\{e\}) \neq \phi$. A subset $C \subseteq \triangle$ is called closed if $B$ contains all its limit points. 
Example 2 (see [6]). Let $\triangle=\mathbb{R}^{+}, a>0$ be any constant and define $\theta_{c b}: \triangle \times \triangle \longrightarrow \mathbb{C}^{+}$by $\theta_{c b}(e, f)=(\max \{e, f\}+a)(1+$ i) $\forall e, f \in \triangle$.

Then, $\left(\triangle, \theta_{c b}\right)$ is a complex partial b-metric space with arbitrary coefficient $s \geq 1$. Now, define a sequence $\left\{e_{n}\right\}$ in $\triangle$ by $e_{n}=1$ for all $n \in \mathbb{N}$. Note that, if $f \geq 1$, we have $\theta_{c b}\left(e_{n}, f\right)=(f+a)(1+i)=\theta_{c b}(f, f)$. Therefore,

$\lim _{n \longrightarrow \infty} \theta_{c b}\left(e_{n}, f\right)=\theta_{c b}(f, f)$ for all $f \geq 1$. Thus, the limit of convergent sequence in complex partial b-metric space need not be unique.

Example 3 (see [6]). Let $\triangle=[0, \infty)$ be endowed with complex partial b-metric $\theta_{c b}: \triangle \times \triangle \longrightarrow \mathbb{C}^{+}$with $\theta_{c b}=(\max \{e, f\})^{2}+i(\max \{e, f\})^{2} \forall e, f \in \triangle$.

It is easy to verify that $\left(\triangle, \theta_{c b}\right)$ is a complex partial b-metric space and note that self-distance need not be zero, for example, $\theta_{c b}(1,1)=1+i \neq 0$. Now, the complex valued b-metric not induced by $\theta_{c b}$ is as follows: $d_{\theta_{c b}}(e, f)=2 \theta_{c b}(e, f)-\theta_{c b}(e, e)-\theta_{c b}(f, f)$; without loss of generality, suppose that $e \geq f$; then, $d_{\theta_{c b}}(e, f)$ $=2\left[(\max \{e, f\})^{2}+i(\max \{e, f\})^{2}\right]-\left(e^{2}+i e^{2}\right)-\left(f^{2}+i f^{2}\right)$. Therefore, $d_{\theta_{c b}}(e, f)=e^{2}-f^{2}+i\left(e^{2}-f^{2}\right)$.

Thus, we have the following proposition.

Proposition 3 (see [6]). There exists a complex partial $b$-metric $\theta_{c b}$ which does not define a complex b-metric $d_{\theta_{c b}}$, where

$d_{\theta_{c b}}(e, f)=2 \theta_{c b}(e, f)-\theta_{c b}(e, e)-\theta_{c b}(f, f) \forall e, f \in \triangle$.

Definition 5 (see [13]). Let $(\triangle, \preceq)$ be a partially ordered set and $\Lambda: \triangle \times \triangle \longrightarrow \triangle$. We say that $\Lambda$ has the mixed monotone property if $\Lambda(i, j)$ is monotone nondecreasing in $i$ and is monotone nonincreasing in $j$, that is, for any $i, j \in \triangle$,

$$
\begin{array}{ll}
i_{1}, i_{2} \in \triangle, & i_{1} \prec i_{2} \Longrightarrow \Lambda\left(i_{1}, j\right) \preceq \Lambda\left(i_{2}, j\right), \\
j_{1}, j_{2} \in \triangle, & j_{1} \prec j_{2} \Longrightarrow \Lambda\left(i, j_{1}\right) \geqslant \Lambda\left(i, j_{2}\right) .
\end{array}
$$

Definition 6. Let $\left(\triangle, \theta_{c b}\right)$ be a complex partial b-metric space. An element $(i, j) \in \triangle \times \triangle$, is called a coupled fixed point of the mapping $\Lambda: \triangle \times \triangle \longrightarrow \triangle$ if $\Lambda(i, j)=i$ and $\Lambda(j, i)=j$.

Example 4. Let $\triangle=[0, \infty)$ be endowed with complex partial b-metric $\theta_{c b}: \triangle \times \triangle \longrightarrow \mathbb{C}^{+}$defined by $\theta_{c b}(e, j)=(\max \{e, j\})^{2}+i(\max \{e, j\})^{2} \forall e, j \in \triangle$. Consider the mapping $\Lambda: \triangle \times \triangle \longrightarrow \triangle$ with $\Lambda(e, j)=i((e+j) / 4)$. Here, $(0,0)$ is the coupled fixed point of $\Lambda$.

In 2019, Gunaseelan and Mishra [14] proved the following theorem.

Theorem 1 (see [14]). Let $\left(U, \xi_{c}\right)$ be a complete complex partial metric space. Suppose that the mapping $\phi: U \times U \longrightarrow U$ satisfies the following contractive condition for all $\alpha, \beta, \gamma, \delta \in U$ :

$$
\xi_{c}(\phi(\alpha, \beta), \phi(\gamma, \delta)) \prec k \xi_{c}(\phi(\alpha, \beta), \alpha)+l \xi_{c}(\phi(\gamma, \delta), \gamma),
$$

where $k, l$ are nonnegative constants with $k+l<1$. Then, $\phi$ has a unique coupled fixed point.

Inspired by Theorem 1, we prove coupled fixed-point theorem on partially ordered complex partial b-metric space using mixed monotone property.

In the next section, we firstly prove that a continuous mapping having the mixed monotone property on a partially ordered complete complex partial b-metric space has a coupled fixed point under certain conditions. Secondly, we give result of a coupled fixed point for a mapping having the mixed monotone property on a partially ordered complete complex partial b-metric space by losing the property of continuity. Then, we find the condition under which a continuous mapping having the mixed monotone property on a partially ordered complete complex partial b-metric space has a unique coupled fixed point under certain conditions. We also give an example of continuous mapping having the mixed monotone property on a partially ordered complete complex partial b-metric space and show that it has unique coupled fixed point under said conditions.

\section{Main Results}

Let $(\triangle, \prec)$ be a partially ordered set and $\theta_{c b}$ be a complex partial b-metric space on $\triangle$. Further, we endow the product space $\triangle \times \triangle$ with the following partial order:

$$
\text { for } \begin{aligned}
&(i, j),(g, h) \in \triangle \times \triangle, \\
&(g, h) \preceq(i, j) \Longleftrightarrow i \geqslant g, j \preceq h .
\end{aligned}
$$

We begin with the following theorem that establishes the existence of a fixed-point theorem for a function $\Lambda$ on the product $\triangle \times \triangle$.

Theorem 2. Let $\left(\triangle, \theta_{c b}, \preceq\right)$ be a partially ordered complete complex partial $b$-metric space with the coefficient $s \geq 1$. Let $\Lambda: \triangle \times \triangle \longrightarrow \triangle$ be a continuous mapping having the mixed monotone property on $\triangle$. Assume that there exists a $2 s \alpha \in[0,1)$ with

$$
\theta_{c b}(\Lambda(i, j), \Lambda(g, h)) \prec \alpha\left[\theta_{c b}(i, g)+\theta_{c b}(j, h)\right], \quad \forall i \geqslant g, j \preceq h .
$$

If there exists $i_{0}, j_{0} \in \triangle$ such that

$$
\begin{aligned}
& i_{0} \preceq \Lambda\left(i_{0}, j_{0}\right), \\
& j_{0} \geqslant \Lambda\left(j_{0}, i_{0}\right),
\end{aligned}
$$

then $\Lambda$ has a coupled fixed point.

Proof. Since $i_{0} \prec \Lambda\left(i_{0}, j_{0}\right)=i_{1}$ (say) and $j_{0} \geqslant \Lambda\left(j_{0}, i_{0}\right)=j_{1}$ (say), letting $i_{2}=\Lambda\left(i_{1}, j_{1}\right)$ and $j_{2}=\Lambda\left(j_{1}, i_{1}\right)$, we denote

$$
\begin{aligned}
& \Lambda^{2}\left(i_{0}, j_{0}\right)=\Lambda\left(\Lambda\left(i_{0}, j_{0}\right), \Lambda\left(j_{0}, i_{0}\right)\right)=\Lambda\left(i_{1}, j_{1}\right)=i_{2}, \\
& \Lambda^{2}\left(j_{0}, i_{0}\right)=\Lambda\left(\Lambda\left(j_{0}, i_{0}\right), \Lambda\left(i_{0}, j_{0}\right)\right)=\Lambda\left(j_{1}, i_{1}\right)=j_{2} .
\end{aligned}
$$

Due to the mixed monotone property of $\Lambda$, 


$$
\begin{aligned}
& i_{2}=\Lambda^{2}\left(i_{0}, j_{0}\right)=\Lambda\left(i_{1}, j_{1}\right) \geqslant \Lambda\left(i_{0}, j_{0}\right)=i_{1}, \\
& j_{2}=\Lambda^{2}\left(j_{0}, i_{0}\right)=\Lambda\left(j_{1}, i_{1}\right) \leq \Lambda\left(j_{0}, i_{0}\right)=j_{1} .
\end{aligned}
$$

Further, for $p=1,2, \ldots$, we let

$$
\begin{aligned}
& i_{p+1}=\Lambda^{p+1}\left(i_{0}, j_{0}\right)=\Lambda\left(\Lambda^{p}\left(i_{0}, j_{0}\right), \Lambda^{p}\left(j_{0}, i_{0}\right)\right), \\
& j_{p+1}=\Lambda^{p+1}\left(j_{0}, i_{0}\right)=\Lambda\left(\Lambda^{p}\left(j_{0}, i_{0}\right), \Lambda^{p}\left(i_{0}, j_{0}\right)\right) .
\end{aligned}
$$

We can easily verify that

$$
i_{0} \preceq \Lambda\left(i_{0}, j_{0}\right)=i_{1} \preceq \Lambda^{2}\left(i_{0}, j_{0}\right)=i_{2} \preceq \cdots \preceq \Lambda^{p+1}\left(i_{0}, j_{0}\right) \preceq \cdots,
$$

$$
j_{0} \geqslant \Lambda\left(j_{0}, i_{0}\right)=j_{1} \geqslant \Lambda^{2}\left(j_{0}, i_{0}\right)=j_{2} \geqslant \cdots \geqslant \Lambda^{p+1}\left(j_{0}, i_{0}\right) \geqslant \cdots .
$$

If $i_{p+1}=i_{p}$ and $j_{p+1}=j_{p}$ for some $p$, then $\Lambda\left(i_{p}, j_{p}\right)=i_{p}$ and $\Lambda\left(j_{p}, i_{p}\right)=j_{p}$, and hence $\left(i_{p}, j_{p}\right)$ is a coupled fixed point of $\Lambda$. Suppose, further, that

$$
\begin{aligned}
& i_{p} \neq i_{p+1}, \\
& \text { or } j_{p} \neq j_{p+1}, \quad \text { for each } p \in \mathbb{N}_{0} .
\end{aligned}
$$

Now, we claim that, for $p \in \mathbb{N}_{0}$,

$\left|\theta_{c b}\left(i_{p+1}, i_{p}\right)\right|+\left|\theta_{c b}\left(j_{p+1}, j_{p}\right)\right| \leq 2^{p} \alpha^{p}\left[\left|\theta_{c b}\left(i_{1}, i_{0}\right)\right|+\left|\theta_{c b}\left(j_{1}, j_{0}\right)\right|\right]$.
Indeed, for $p=1$, using $i_{1} \geqslant i_{0}, j_{1} \preceq j_{0}$, we get

$$
\begin{aligned}
\theta_{c b}\left(i_{2}, i_{1}\right) & =\theta_{c b}\left(\Lambda\left(i_{1}, j_{1}\right), \Lambda\left(i_{0}, j_{0}\right)\right) \\
& \preceq \alpha\left[\theta_{c b}\left(i_{1}, i_{0}\right)+\theta_{c b}\left(j_{1}, j_{0}\right)\right],
\end{aligned}
$$

which implies that

$$
\left|\theta_{c b}\left(i_{2}, i_{1}\right)\right| \leq \alpha\left[\left|\theta_{c b}\left(i_{1}, i_{0}\right)\right|+\left|\theta_{c b}\left(j_{1}, j_{0}\right)\right|\right] .
$$

Similarly,

$$
\begin{aligned}
\theta_{c b}\left(j_{2}, j_{1}\right) & =\theta_{c b}\left(\Lambda\left(j_{1}, i_{1}\right), \Lambda\left(j_{0}, i_{0}\right)\right) \\
& \preceq \alpha\left[\theta_{c b}\left(j_{1}, j_{0}\right)+\theta_{c b}\left(i_{1}, i_{0}\right)\right],
\end{aligned}
$$

which implies that

$$
\left|\theta_{c b}\left(j_{2}, j_{1}\right)\right| \leq \alpha\left[\left|\theta_{c b}\left(j_{1}, j_{0}\right)\right|+\left|\theta_{c b}\left(i_{1}, i_{0}\right)\right|\right] .
$$

Adding (12) and (16), we have

$$
\left|\theta_{c b}\left(i_{2}, i_{1}\right)\right|+\left|\theta_{c b}\left(j_{2}, j_{1}\right)\right| \leq 2 \alpha\left[\left|\theta_{c b}\left(i_{0}, i_{1}\right)\right|+\left|\theta_{c b}\left(j_{0}, j_{1}\right)\right|\right] .
$$

In a similar way, proceeding by induction, if we assume that (12) holds, we get that

$$
\begin{aligned}
\left|\theta_{c b}\left(i_{p+2}, i_{p+1}\right)\right|+\left|\theta_{c b}\left(j_{p+2}, j_{p+1}\right)\right| & \leq 2 \alpha\left[\left|\theta_{c b}\left(i_{p+1}, i_{p}\right)\right|+\left|\theta_{c b}\left(j_{p+1}, j_{p}\right)\right|\right] \\
& \leq 2^{p+1} \alpha^{p+1}\left[\left|\theta_{c b}\left(i_{0}, i_{1}\right)\right|+\theta_{c b}\left(j_{0}, j_{1}\right)\right] . \\
& l_{p} \leq 2^{p} \alpha^{p} l_{0} .
\end{aligned}
$$

Hence, by induction, (12) is proved. Set

$$
l_{p}:=\left|\theta_{c b}\left(i_{p}, i_{p+1}\right)\right|+\left|\theta_{c b}\left(j_{p}, j_{p+1}\right)\right|, \quad p \in \mathbb{N} .
$$

By assumption (9), $l_{p}>0$ for $p \in \mathbb{N}_{0}$. Then, for each

Then, the sequence $\left\{l_{p}\right\}$ is decreasing and $p \geq q$, we have

$$
\begin{array}{rl}
\theta_{c b}\left(i_{q}, i_{p}\right) \leq & s \theta_{c b}\left(i_{q}, i_{q+1}\right)+s^{2} \theta_{c b}\left(i_{q+1}, i_{q+2}\right)+\cdots+s^{p} \theta_{c b}\left(i_{p-1}, i_{p}\right) \\
& -\theta_{c b}\left(i_{q+1}, i_{q+1}\right)-\theta_{c b}\left(i_{q+2}, i_{q+2}\right)-\theta_{c b}\left(i_{q+3}, i_{q+3}\right) \\
& -\cdots-\theta_{c b}\left(i_{p-1}, i_{p-1}\right) \\
\leq & s \theta_{c b}\left(i_{q}, i_{q+1}\right)+s^{2} \theta_{c b}\left(i_{q+1}, i_{q+2}\right)+\cdots+s^{p} \theta_{c b}\left(i_{p-1}, i_{p}\right), \\
\theta_{c b}\left(j_{q}, j_{p}\right) \leq s & s \theta_{c b}\left(j_{q}, j_{q+1}\right)+s^{2} \theta_{c b}\left(j_{q+1}, j_{q+2}\right)+\cdots+s^{p} \theta_{c b}\left(j_{p-1}, j_{p}\right) \\
& -\theta_{c b}\left(j_{q+1}, j_{q+1}\right)-\theta_{c b}\left(j_{q+2}, j_{q+2}\right)-\theta_{c b}\left(j_{q+3}, j_{q+3}\right) \\
& -\cdots-\theta_{c b}\left(j_{p-1}, j_{p-1}\right) \\
\leq & s \theta_{c b}\left(j_{q}, j_{q+1}\right)+s^{2} \theta_{c b}\left(j_{q+1}, j_{q+2}\right)+\cdots+s^{p} \theta_{c b}\left(j_{p-1}, j_{p}\right),
\end{array}
$$


which implies that

$$
\begin{gathered}
\left|\theta_{c b}\left(i_{q}, i_{p}\right)\right| \leq s\left|\theta_{c b}\left(i_{q}, i_{q+1}\right)\right|+s^{2}\left|\theta_{c b}\left(i_{q+1}, i_{q+2}\right)\right|+\cdots+s^{p}\left|\theta_{c b}\left(i_{p-1}, i_{p}\right)\right|, \\
\left|\theta_{c b}\left(j_{q}, j_{p}\right)\right| \leq s\left|\theta_{c b}\left(j_{q}, j_{q+1}\right)\right|+s^{2}\left|\theta_{c b}\left(j_{q+1}, j_{q+2}\right)\right|+\cdots+s^{p}\left|\theta_{c b}\left(j_{p-1}, j_{p}\right)\right| .
\end{gathered}
$$

Therefore,

$$
\begin{aligned}
l_{q}=\left|\theta_{c b}\left(i_{q}, i_{p}\right)\right|+\left|\theta_{c b}\left(j_{q}, j_{p}\right)\right| \leq & s\left(\left|\theta_{c b}\left(i_{q}, i_{q+1}\right)\right|+\left|\theta_{c b}\left(j_{q}, j_{q+1}\right)\right|\right) \\
& +s^{2}\left(\left|\theta_{c b}\left(i_{q+1}, i_{q+2}\right)\right|+\theta_{c b}\left(j_{q+1}, j_{q+2}\right) \mid\right)+\cdots \\
& \cdots+s^{p}\left(\theta_{c b}\left(i_{p-1}, i_{p}\right)\left|+\theta_{c b}\left(j_{p-1}, j_{p}\right)\right|\right) \\
\leq & s l_{q}+s^{2} l_{q+1}+\cdots+s^{p} l_{p-1} \\
\leq & s 2^{q} \alpha^{q}\left(1+s 2 \alpha+\cdots+s^{p-1}(2 \alpha)^{p-q-1}\right) l_{0} \\
\leq & \frac{s(2 \alpha)^{q}}{1-s 2 \alpha} l_{0} \longrightarrow 0, \quad \text { as } q \longrightarrow \infty .
\end{aligned}
$$

Therefore, $\left\{i_{p}\right\}$ and $\left\{j_{p}\right\}$ are Cauchy sequences in $\triangle$. Since $\triangle$ is complete complex partial b-metric space, there exists $(t, r) \in \triangle \times \triangle$ such that

$$
\begin{aligned}
& \lim _{p \longrightarrow \infty} i_{p}=t, \\
& \lim _{p \longrightarrow \infty} j_{p}=r,
\end{aligned}
$$

and $\theta_{c b}(t, t)=\lim _{p \longrightarrow \infty} \theta_{c b}\left(t, t_{p}\right)=\lim _{p, q \longrightarrow \infty} \theta_{c b}\left(t_{p}, t_{q}\right)=0$ and $\theta_{c b}(r, r)=\lim _{p \longrightarrow \infty} \theta_{c b}\left(r, r_{p}\right)=\lim _{p, q \longrightarrow \infty} \theta_{c b}\left(r_{p}, r_{q}\right)$ $=0$. Finally, we claim that $(t, r)$ is a coupled fixed point of $\Lambda$. Indeed, from $i_{p+1}=\Lambda\left(i_{p}, j_{p}\right)$ and $j_{p+1}=\Lambda\left(j_{p}, i_{p}\right)$, using (24) and the continuity of $\Lambda$, it immediately follows that $t=$ $\Lambda(t, r)$ and $r=\Lambda(r, t)$.

In the next theorem, we will substitute the continuity hypothesis on $\Lambda$ by an additional property satisfied by the space $\left(\triangle, \theta_{c b}, \prec\right)$.

Theorem 3. Let $\left(\triangle, \theta_{c b}, \prec\right)$ be a partially ordered complete complex partial b-metric space with the coefficient $s \geq 1$. Let $\Lambda: \triangle x \triangle \longrightarrow \triangle$ be a mapping having the mixed monotone property on $\triangle$. Assume that there exists $2 s \alpha \in[0,1)$ with

$\theta_{c b}(\Lambda(i, j), \Lambda(g, h))<\alpha\left[\theta_{c b}(i, g)+\theta_{c b}(j, h)\right], \quad \forall i \geqslant g, j \leq h$.

Finally, assume that $\triangle$ has the following properties:

(i) If a nondecreasing sequence $\left\{i_{p}\right\}$ in $\triangle$ converges to $i \in \triangle$, then $i_{p}<i$ for all $p$. (ii) If a nonincreasing sequence $\left\{j_{p}\right\}$ in $\triangle$ converges to $j \in \triangle$, then $j_{p} \geqslant j$ for all $p$.

Then, $\Lambda$ has a coupled fixed point.

Proof. Following the proof of Theorem 2, we only have to show $(t, r)$ is a coupled fixed point of $\Lambda$. We have

$$
\begin{aligned}
\theta_{c b}(\Lambda(t, r), t) \leq & s\left(\theta_{c b}\left(\Lambda(t, r), i_{p+1}\right)+\theta_{c b}\left(i_{p+1}, t\right)\right) \\
& -\theta_{c b}\left(i_{p+1}, i_{p+1}\right) \\
\leq & s\left(\theta_{c b}\left(\Lambda(t, r), i_{p+1}\right)+\theta_{c b}\left(i_{p+1}, t\right)\right) \\
= & s\left(\theta_{c b}\left(\Lambda(t, r), i_{p+1}\right)+\theta_{c b}\left(i_{p+1}, t\right)\right) .
\end{aligned}
$$

Since the nondecreasing sequence $\left\{i_{p}\right\}$ converges to $t$ and the nonincreasing sequence $\left\{j_{p}\right\}$ converges to $r$, by (i)-(iii), we have

$$
\begin{aligned}
& t \geqslant i_{p}, \\
& r \preceq j_{p}, \quad \forall p .
\end{aligned}
$$

Now, from the contractive condition (25), we have

$$
\theta_{c b}\left(\Lambda(t, r), \Lambda\left(i_{p}, j_{p}\right)\right) \prec \alpha\left(\theta_{c b}\left(t, i_{p}\right)+\theta_{c b}\left(r, j_{p}\right)\right) .
$$

Then, from (26), we get

$$
\theta_{c b}(\Lambda(t, r), t) \preceq s\left(\alpha\left(\theta_{c b}\left(t, i_{p}\right)+\theta_{c b}\left(r, j_{p}\right)\right)+\theta_{c b}\left(i_{p+1}, t\right)\right),
$$

which implies that 


$$
\left|\theta_{c b}(\Lambda(t, r), t)\right| \leq s \alpha\left|\theta_{c b}\left(t, i_{p}\right)\right|+s \alpha\left|\theta_{c b}\left(r, j_{p}\right)\right|+s\left|\theta_{c b}\left(i_{p+1}, t\right)\right| .
$$

Taking limit as $p \longrightarrow \infty$, we have

$$
\left|\theta_{c b}(\Lambda(t, r), t)\right| \leq 0
$$

Therefore, $\Lambda(t, r)=t$. Similarly, we can prove that $\Lambda(r, t)=r$. Hence, $(t, r)$ is a coupled fixed point of $\Lambda$.

Theorem 4. Assume that

$$
\begin{array}{r}
\forall(e, f),\left(e^{*}, f^{*}\right) \in \triangle \times \triangle, \\
\exists\left(x_{1}, x_{2}\right) \in \triangle \times \triangle
\end{array}
$$

that is comparable to $(e, f)$ and $\left(e^{*}, f^{*}\right)$.

Adding (32) to the hypotheses of Theorem 2, we obtain the uniqueness of the coupled fixed point of $\Lambda$.

Proof. From Theorem 2, we know that there exists a coupled fixed point $(t, r)$ of $\Lambda$, which is obtained as $t=\lim _{p \longrightarrow \infty} \Lambda^{p}\left(i_{0}, j_{0}\right)$ and $r=\lim _{p \longrightarrow \infty} \Lambda^{p}\left(j_{0}, i_{0}\right)$. Suppose that $\left(e^{*}, f^{*}\right)$ is another coupled fixed point, i.e.,

$$
\begin{aligned}
& \Lambda\left(e^{*}, f^{*}\right)=e^{*}, \\
& \Lambda\left(f^{*}, e^{*}\right)=f^{*} .
\end{aligned}
$$

Let us claim that

$$
\theta_{c b}\left(t, e^{*}\right)+\theta_{c b}\left(r, f^{*}\right)=0 .
$$

We discuss two cases.

Case 1: $(t, r)$ is comparable with $\left(e^{*}, f^{*}\right)$ with respect to the ordering in $\triangle \times \triangle$. Let, e.g., $t \geqslant e^{*}$ and $r \prec f^{*}$.
Then, we can apply the contractive condition (4) to obtain

$$
\begin{aligned}
\theta_{c b}\left(t, e^{*}\right) & =\theta_{c b}\left(\Lambda(t, r), \Lambda\left(e^{*}, f^{*}\right)\right) \\
& \preceq \alpha\left[\theta_{c b}\left(t, e^{*}\right)+\theta_{c b}\left(r, f^{*}\right)\right],
\end{aligned}
$$

which implies that

$$
\begin{aligned}
\left|\theta_{c b}\left(t, e^{*}\right)\right| & \leq \alpha\left[\left|\theta_{c b}\left(t, e^{*}\right)\right|+\left|\theta_{c b}\left(r, f^{*}\right)\right|\right] . \\
\theta_{c b}\left(r, f^{*}\right) & =\theta_{c b}\left(\Lambda(r, t), \Lambda\left(f^{*}, e^{*}\right)\right) \\
& \leq \alpha\left[\theta_{c b}\left(r, f^{*}\right)+\theta_{c b}\left(t, e^{*}\right)\right],
\end{aligned}
$$

which implies that

$$
\left|\theta_{c b}\left(r, f^{*}\right)\right| \leq \alpha\left[\left|\theta_{c b}\left(r, f^{*}\right)\right|+\left|\theta_{c b}\left(t, e^{*}\right)\right|\right] .
$$

Adding (36) and (38), we get

$$
\left|\theta_{c b}\left(t, e^{*}\right)\right|+\left|\theta_{c b}\left(r, f^{*}\right)\right| \leq 2 \alpha\left[\left|\theta_{c b}\left(t, e^{*}\right)\right|+\left|\theta_{c b}\left(r, f^{*}\right)\right|\right] .
$$

Since $2 \alpha \in[0,(1 / s))$, (34) holds.

Case 2: $(t, r)$ is not comparable with $\left(e^{*}, f^{*}\right)$. In this case, there exists $\left(x_{1}, x_{2}\right) \in \triangle \times \triangle$ that is comparable both to $(t, r)$ and $\left(e^{*}, f^{*}\right)$. Then, for all $p \in \mathbb{N},\left(\Lambda^{p}\left(x_{1}\right.\right.$, $\left.\left.x_{2}\right), \Lambda^{p}\left(x_{2}, x_{1}\right)\right)$ is comparable both to $\left(\Lambda^{p}(t, r), \Lambda^{p}(r\right.$, $t))=(t, r)$ and $\left(\Lambda^{p}\left(e^{*}, f^{*}\right), \Lambda^{p}\left(f^{*}, e^{*}\right)\right)=\left(e^{*}, f^{*}\right)$. We have

$$
\begin{aligned}
\theta_{c b}\left(t, e^{*}\right)+\theta_{c b}\left(r, f^{*}\right)= & \theta_{c b}\left(\Lambda^{p}(t, r), \Lambda^{p}\left(e^{*}, f^{*}\right)\right)+\theta_{c b}\left(\Lambda^{p}(r, t), \Lambda^{p}\left(f^{*}, e^{*}\right)\right) \\
\preceq & \theta_{c b}\left(\Lambda^{p}(t, r), \Lambda^{p}\left(x_{1}, x_{2}\right)\right)+\theta_{c b}\left(\Lambda^{p}\left(x_{1}, x_{2}\right), \Lambda^{p}\left(e^{*}, f^{*}\right)\right) \\
& +\theta_{c b}\left(\Lambda^{p}(r, t), \Lambda^{p}\left(x_{2}, x_{1}\right)\right)+\theta_{c b}\left(\Lambda^{p}\left(x_{2}, x_{1}\right), \Lambda^{p}\left(f^{*}, e^{*}\right)\right) \\
\preceq & 2^{p} \alpha^{p}\left[\theta_{c b}\left(t, x_{1}\right)+\theta_{c b}\left(r, x_{2}\right)+\theta_{c b}\left(e^{*}, x_{1}\right)+\theta_{c b}\left(f^{*}, x_{2}\right)\right],
\end{aligned}
$$

which implies that

$$
\begin{array}{r}
\left|\theta_{c b}\left(t, e^{*}\right)\right|+\left|\theta_{c b}\left(r, f^{*}\right)\right| \leq \\
2^{p} \alpha^{p}\left[\left|\theta_{c b}\left(t, x_{1}\right)\right|+\left|\theta_{c b}\left(r, x_{2}\right)\right|\right. \\
\left.+\left|\theta_{c b}\left(e^{*}, x_{1}\right)\right|+\left|\theta_{c b}\left(f^{*}, x_{2}\right)\right|\right] .
\end{array}
$$

Since $2 \alpha \in[0,(1 / s)),(34)$ holds.

We deduce that in all cases, (34) holds. This implies that $(t, r)=\left(e^{*}, f^{*}\right)$ and the uniqueness of the coupled fixed point of $\Lambda$ is proved.
Theorem 5. In addition to the hypotheses of Theorem 2 (resp. Theorem 3), suppose that $i_{0}, j_{0}$ in $\triangle$ are comparable. Then, $t=r$.

Proof. Suppose that $i_{0} \preceq j_{0}$. We claim that

$$
i_{p} \preceq j_{p}, \quad \forall p \in \mathbb{N} \text {. }
$$

From the mixed monotone property of $\Lambda$, we have

$$
i_{1}=\Lambda\left(i_{0}, j_{0}\right) \preceq \Lambda\left(j_{0}, j_{0}\right) \preceq \Lambda\left(j_{0}, i_{0}\right)=j_{1} .
$$

Assume that $i_{p} \preceq j_{p}$ for some $p$. Now, 


$$
\begin{aligned}
i_{p+1} & =\Lambda^{p+1}\left(i_{0}, j_{0}\right)=\Lambda\left(\Lambda^{p}\left(i_{0}, j_{0}\right), \Lambda^{p}\left(j_{0}, i_{0}\right)\right) \\
& =\Lambda\left(i_{p}, j_{p}\right) \\
& \leq \Lambda\left(j_{p}, j_{p}\right) \preceq \Lambda\left(j_{p}, i_{p}\right) \\
& =j_{p+1} .
\end{aligned}
$$

Hence, (42) holds.

Now, using (42) and the contractive condition, we get

$$
\begin{aligned}
\theta_{c b}(t, r) & \leq s\left(\theta_{c b}\left(t, i_{p+1}\right)+\theta_{c b}\left(i_{p+1}, r\right)\right)-\theta_{c b}\left(i_{p+1}, i_{p+1}\right) \\
& \leq s\left(\theta_{c b}\left(t, i_{p+1}\right)+\theta_{c b}\left(i_{p+1}, r\right)\right) \\
& \leq s\left(\theta_{c b}\left(t, i_{p+1}\right)+s\left(\theta_{c b}\left(i_{p+1}, j_{p+1}\right)+\theta_{c b}\left(j_{p+1}, r\right)-\theta_{c b}\left(j_{p+1}, j_{p+1}\right)\right)\right) \\
& \leq s \theta_{c b}\left(t, i_{p+1}\right)+s^{2} \theta_{c b}\left(i_{p+1}, j_{p+1}\right)+s^{2} \theta_{c b}\left(j_{p+1}, r\right) \\
& =s \theta_{c b}\left(t, i_{p+1}\right)+s^{2}\left(\theta_{c b}\left(\Lambda\left(i_{p}, j_{p}\right), \Lambda\left(j_{p}, i_{p}\right)\right)+s^{2} \theta_{c b}\left(j_{p+1}, r\right)\right. \\
& \leq s \theta_{c b}\left(t, i_{p+1}\right)+s^{2} \alpha\left(\theta_{c b}\left(i_{p}, j_{p}\right)+\theta_{c b}\left(j_{p}, i_{p}\right)\right)+s^{2} \theta_{c b}\left(j_{p+1}, r\right),
\end{aligned}
$$

which implies that

$$
\left|\theta_{c b}(t, r)\right| \leq s\left|\theta_{c b}\left(t, i_{p+1}\right)\right|+s^{2} \alpha\left(\left|\theta_{c b}\left(i_{p}, j_{p}\right)\right|+\left|\theta_{c b}\left(j_{p}, i_{p}\right)\right|\right)+s^{2}\left|\theta_{c b}\left(j_{p+1}, r\right)\right| .
$$

Passing to the limit as $p \longrightarrow \infty$, we get

$$
\theta_{c b}(t, r) \leq 2 s^{2} \alpha \theta_{c b}(t, r) .
$$

Since $2 s^{2} \alpha<1$, this implies that $\theta_{c b}(t, r)=0$, i.e., $t=r$.

Example 5. Let $\triangle=[1, \infty)$ be equipped with the partial order $\leqslant$ defined by

$$
e \prec f \Longleftrightarrow e \leq f,
$$

and with the functional $\theta_{c b}: \triangle \times \triangle \longrightarrow \mathbb{C}^{+}$defined by $\theta_{c b}(e, f)=|e-f|^{2}+2+i\left(|e-f|^{2}+2\right)$ for all $e, f \in \triangle$. Clearly, $\left(\triangle, \theta_{c b}\right)$ is a partially ordered complete complex partial b-metric space with $s=2$. Define the mapping $\Lambda: \triangle \times \triangle \longrightarrow \triangle$ by

$$
\Lambda(e, f)= \begin{cases}0, & \text { if } e<f, \\ \frac{e-f}{2}, & \text { if } e \geq f .\end{cases}
$$

Obviously, the mapping $\Lambda$ has the mixed monotone property and is continuous. Let $e, f, t, r \in \Delta$ be such that $e \leq t$ and $f \geq r$. We have considered the following cases.

Case 1: $e \geq f$. Since $e \leq t$, we have $t \geq e \geq f \geq r$ :

$$
\begin{aligned}
\theta_{c b}(\Lambda(e, f), \Lambda(t, r)) & =\theta_{c b}\left(\frac{e-f}{2}, \frac{t-r}{2}\right) \\
& =\left|\frac{e-f}{2}-\frac{t-r}{2}\right|^{2}+2+i\left(\left|\frac{e-f}{2}-\frac{t-r}{2}\right|^{2}+2\right) \\
& =\left|\frac{e-t}{2}+\frac{r-f}{2}\right|^{2}+2+i\left(\left|\frac{e-t}{2}+\frac{r-f}{2}\right|^{2}+2\right) \\
& \leq 2\left(\left|\frac{e-t}{2}\right|^{2}+\left|\frac{r-f}{2}\right|^{2}\right)+2+i\left(2\left(\left|\frac{e-t}{2}\right|^{2}+\left|\frac{r-f}{2}\right|^{2}\right)+2\right) \\
& =\frac{1}{2}\left(|e-t|^{2}+|r-f|^{2}+4+i\left(|e-t|^{2}+|r-f|^{2}+4\right)\right) \\
& =\alpha\left(\theta_{c b}(e, t)+\theta_{c b}(r, t)\right) .
\end{aligned}
$$


Case 2: $e<f, t \geq r$, and $e>r$ :

$$
\begin{aligned}
\theta_{c b}(\Lambda(e, f), \Lambda(t, r)) & =\left|0-\frac{t-r}{2}\right|^{2}+2+i\left(\left|0-\frac{t-r}{2}\right|^{2}+2\right) \\
& =\frac{|t-r|^{2}}{4}+2+i\left(\frac{|t-r|^{2}}{4}+2\right) \\
& \preceq \frac{|t-r+f-e|^{2}}{4}+2+i\left(\frac{|t-r+f-e|^{2}}{4}+2\right) \\
& \leq \frac{1}{2}\left(|e-t|^{2}+|f-r|^{2}+4\right)+i\left(\frac{1}{2}\left(|e-t|^{2}+|f-r|^{2}+4\right)\right) \\
& =\alpha\left(\theta_{c b}(e, t)+\theta_{c b}(r, t)\right) .
\end{aligned}
$$

Case 3: $e<f, t \geq r$, and $e<r$ :

$$
\begin{aligned}
\theta_{c b}(\Lambda(e, f), \Lambda(t, r) & =\left|0-\frac{t-r}{2}\right|^{2}+2+i\left(\left|0-\frac{t-r}{2}\right|^{2}+2\right) \\
& =\left|\frac{t-r}{2}\right|^{2}+2+i\left(\left|\frac{t-r}{2}\right|^{2}+2\right) \\
& \leq \frac{|t-r+f-e|^{2}}{4}+2+i\left(\frac{|t-r+f-e|^{2}}{4}+2\right) \\
& \leq \frac{1}{2}\left(|e-t|^{2}+|f-r|^{2}+4\right)+i\left(\frac{1}{2}\left(|e-t|^{2}+|f-r|^{2}+4\right)\right) \\
& =\alpha\left(\theta_{c b}(e, t)+\theta_{c b}(r, t)\right) .
\end{aligned}
$$

Case 4: $e<f$ and $t<r$ :

$$
\begin{aligned}
\theta_{c b}(\Lambda(e, f), \Lambda(t, r))= & \theta_{c b}(0,0)=2(1+i) \prec \alpha\left(\theta_{c b}(e, t)\right. \\
& \left.+\theta_{c b}(r, t)\right) .
\end{aligned}
$$

Thus, $\Lambda$ satisfies all assumptions of Theorem 4 and it has a unique coupled fixed point (which is $(0,0)$ ).
Next, we present a result for the existence of a unique solution for a particular system of integral equations.

3.1. Applications to Integral Equations. We study the existence of solutions for the following system of integral equations:

$$
\begin{aligned}
& e(u)=\int_{a}^{b}\left(T_{1}(u, s)+T_{2}(u, s)\right)(H(s, e(s))+K(s, f(s))) \mathrm{d} s+l(u), \\
& f(u)=\int_{a}^{b}\left(T_{1}(u, s)+T_{2}(u, s)\right)(H(s, f(s))+K(s, e(s))) \mathrm{d} s+l(u),
\end{aligned}
$$

where $u \in I=[a, b]$.

We assume that $T_{1}, T_{2}, H, K$ satisfy the following conditions: (i) $T_{1}(u, s) \geqslant 0$ and $T_{2}(u, s) \prec 0$ for all $u, s \in[a, b]$.

(ii) There exist $e, f \in \mathbb{R}, e \prec f$ such that 


$$
\begin{aligned}
& 0 \preccurlyeq H(u, e)-H(u, f) \preccurlyeq(e-f) \\
& \text { and }-(e-f) \preccurlyeq K(u, e)-K(u, f) \preccurlyeq 0 . \\
& \text { (iii) } \int_{a}^{b}\left|T_{1}(u, s)-T_{2}(u, s)\right|^{2} \mathrm{~d} s \preceq \alpha / 4 .
\end{aligned}
$$

Theorem 6. Consider integral equations (54) and (55) with $T_{1}, T_{2} \in C(I, \mathbb{R}), H, K \in C(I \times \mathbb{R}, \mathbb{R})$, and $l \in C(I, \mathbb{R})$. Under assumptions (i)-(iii), equations (54) and (55) have a unique solution.

Proof. Consider the natural order relation on $\triangle=C(I, \mathbb{R})$; that is, for $e, f \in C(I, \mathbb{R})$,

$$
e \prec f \Longleftrightarrow e(u) \leq f(u), \quad \forall u \in I .
$$

It is well known that $\triangle$ is a complete complex partial b-metric space with respect to

$$
\theta_{c b}(e, f)=|e-f|^{2}+2+i\left(|e-f|^{2}+2\right), \quad e, f \in C(I, \mathbb{R}) .
$$

Suppose that $\left\{t_{p}\right\}$ is a monotone nondecreasing sequence in $\triangle$ that converges to a point $t \in \triangle$. Then, for every $u \in I$, the sequence of real numbers

$$
t_{1}(u) \leq t_{2}(u) \leq \cdots \leq t_{p}(u) \leq \cdots
$$

converges to $t(u)$. Therefore, for all $u \in I, p \in \mathbb{N}, t_{p} \leq t(u)$. Hence, $t_{p} \leq t$, for all $p$. Similarly, it can be verified that, if for all $u \in I, r(u)$ is a limit of a monotone nondecreasing sequence $\left\{r_{p}\right\}$ in $\triangle$, then $r(u) \leq r_{p}(u)$ for all $p$, and hence $r \leq r_{p}$ for all $p$.

Also, $\triangle \times \triangle=C(I, \mathbb{R}) \times C(I, \mathbb{R})$ is a partially ordered set under the following order relation in $\triangle \times \triangle$ :

$$
\begin{aligned}
(e, f),(t, r) \in \Delta \times \triangle & \\
(e, f) \leq(t, r) & \Longleftrightarrow e(u) \prec t(u), \\
f(u) & \geqslant r(u), \quad \forall u \in I .
\end{aligned}
$$

For any $e, f \in \triangle, \max \{e(u), f(u)\}$ and $\min \{e(u), f(u)\}$, for each $u \in I$, are in $\triangle$ and are the upper and lower bounds of $e, f$, respectively. Therefore, for every $(e, f),(t, r) \in \triangle$ $\times \triangle$, there exists $(\max \{e, t\}, \min \{f, r\}) \in \triangle \times \triangle$ that is comparable to $(e, f)$ and $(t, r)$. Define $\Lambda: \triangle \times \triangle \longrightarrow \triangle$ by

$$
\Lambda(e, f)(u)=\int_{a}^{b} T_{1}(u, s)(H(s, e(s))+K(s, f(s))) \mathrm{d} s+\int_{a}^{b} T_{2}(u, s)(H(s, f(s))+K(s, e(s))) \mathrm{d} s+l(u), \quad \text { for all } u \in[a, b]
$$

We now claim that $\Lambda$ has the mixed monotone property.

If $\left(t_{1}, r\right) \prec\left(t_{2}, r\right)$, then

$$
\begin{aligned}
\Lambda\left(t_{1}, r\right)(u) & =\int_{a}^{b} T_{1}(u, s)\left(H\left(s, t_{1}(s)\right)+K(s, r(s))\right) \mathrm{d} s+\int_{a}^{b} T_{2}(u, s)\left(H(s, r(s))+K\left(s, t_{1}(s)\right)\right) \mathrm{d} s+l(u) \\
& \preceq \int_{a}^{b} T_{1}(u, s)\left(H\left(s, t_{2}(s)\right)+K(s, r(s))\right) \mathrm{d} s+\int_{a}^{b} T_{2}(u, s)\left(H(s, r(s))+K\left(s, t_{2}(s)\right)\right) \mathrm{d} s+l(u) \\
& =\Lambda\left(t_{2}, r\right)(u) .
\end{aligned}
$$

Similarly, if $\left(t, r_{1}\right) \preceq\left(t, r_{2}\right)$, then

$$
\begin{aligned}
\Lambda\left(t, r_{1}\right)(u)= & \int_{a}^{b} T_{1}(u, s)\left(H(s, t(s))+K\left(s, r_{1}(s)\right)\right) \mathrm{d} s \\
& +\int_{a}^{b} T_{2}(u, s)\left(H\left(s, r_{1}(s)\right)+K(s, t(s))\right) \mathrm{d} s+l(u) \\
& \preceq \int_{a}^{b} T_{1}(u, s)\left(H(s, t(s))+K\left(s, r_{2}(s)\right)\right) \mathrm{d} s \\
& +\int_{a}^{b} T_{2}(u, s)\left(H\left(s, r_{2}(s)\right)+K(s, t(s))\right) \mathrm{d} s+l(u) \\
= & \Lambda\left(t, r_{2}\right)(u) .
\end{aligned}
$$


Thus, $\Lambda(t, r)$ is monotone nondecreasing in $t$ and $\Lambda(t, r)$ is monotone nonincreasing in $r$. Also, for $(e, f) \preceq(t, r)$, that is, $t \geqslant e, r \prec f$, it follows that

$$
\begin{aligned}
\theta_{c b}(\Lambda(t, r), \Lambda(e, f))= & |\Lambda(t, r)-\Lambda(e, f)|^{2}+2+i\left(|\Lambda(t, r)-\Lambda(e, f)|^{2}+2\right) \\
= & \mid \int_{a}^{b} T_{1}(u, s)(H(s, t(s))+K(s, r(s))) \mathrm{d} s+\int_{a}^{b} T_{2}(u, s)(H(s, r(s))+K(s, t(s))) \mathrm{d} s+l(u) \\
& -\int_{a}^{b} T_{1}(u, s)(H(s, e(s))+K(s, f(s))) \mathrm{d} s-\int_{a}^{b} T_{2}(u, s)(H(s, f(s))+K(s, e(s))) \mathrm{d} s-\left.l(u)\right|^{2}+2 \\
& +i\left(\mid \int_{a}^{b} T_{1}(u, s)(H(s, t(s))+K(s, r(s))) \mathrm{d} s+T_{2}(u, s)(H(s, r(s))+K(s, t(s))) \mathrm{d} s+l(u)\right. \\
& \left.-\int_{a}^{b} T_{1}(u, s)(H(s, e(s))+K(s, f(s))) \mathrm{d} s-\int_{a}^{b} T_{2}(u, s)(H(s, f(s))+K(s, e(s))) \mathrm{d} s-\left.l(u)\right|^{2}+2\right)
\end{aligned}
$$$$
\theta_{c b}(\Lambda(t, r), \Lambda(e, f))=\mid \int_{a}^{b} T_{1}(u, s)(H(s, t(s))-H(s, e(s))+K(s, r(s))-K(s, f(s))) \mathrm{d} s
$$$$
-\left.\int_{a}^{b} T_{2}(u, s)(H(s, f(s))-H(s, r(s))+K(s, e(s))-K(s, t(s)))\right|^{2}+2
$$$$
+i\left(\mid \int_{a}^{b} T_{1}(u, s)(H(s, t(s))-H(s, e(s))+K(s, r(s))-K(s, f(s))) \mathrm{d} s\right.
$$$$
\left.-\left.\int_{a}^{b} T_{2}(u, s)(H(s, f(s))-H(s, r(s))+K(s, e(s))-K(s, t(s)))\right|^{2}+2\right)
$$$$
\preceq\left|\int_{a}^{b} T_{1}(u, s)(t(s)-e(s)+f(s)-r(s)) \mathrm{d} s-\int_{a}^{b} T_{2}(u, s)(f(s)-r(s)+t(s)-e(s)) \mathrm{d} s\right|^{2}+2
$$$$
+i\left(\left|\int_{a}^{b} T_{1}(u, s)(t(s)-e(s)+f(s)-r(s)) \mathrm{d} s-\int_{a}^{b} T_{2}(u, s)(f(s)-r(s)+t(s)-e(s)) \mathrm{d} s\right|^{2}+2\right)
$$$$
\preceq 4 \int_{a}^{b}\left|T_{1}(u, s)-T_{2}(u, s)\right|^{2}\left(|f(s)-r(s)|^{2}+|t(s)-e(s)|^{2}\right) \mathrm{d} s+4
$$$$
+i\left(4 \int_{a}^{b}\left|T_{1}(u, s)-T_{2}(u, s)\right|^{2}\left(|f(s)-r(s)|^{2}+|t(s)-e(s)|^{2}\right) \mathrm{d} s+4\right)
$$$$
\preceq \frac{4 \alpha}{4}\left(\theta_{c b}(f, r)+\theta_{c b}(t, e)\right)
$$$$
=\alpha\left(\theta_{c b}(f, r)+\theta_{c b}(t, e)\right) .
$$ 
Now, all the hypotheses of Theorem 4 are satisfied. Therefore, $\Lambda$ has a unique coupled fixed point.

\section{Conclusion}

In 2019, Gunaseelan and Mishra [14] proved coupled fixedpoint theorem on complex partial metric space. In this paper, we proved coupled fixed-point theorems on complex partial b-metric space using partially ordered set and mixed monotone property. An illustrative application in partially ordered complex partial b-metric space is given.

\section{Data Availability}

No data were used to support the study.

\section{Conflicts of Interest}

The authors declare that they have no conflicts of interest.

\section{Authors' Contributions}

All authors contributed equally in preparation of this paper. All authors read and approved the final manuscript.

\section{Acknowledgments}

This study was supported by the Higher Education Commission of Pakistan and the Basque Government (grant no. IT1207-19).

\section{References}

[1] I. A. Bakhtin, "The contraction mappings principle in quasimetric spaces," Functional Analysis, vol. 30, pp. 26-37, 1989, in Russian.

[2] S. Czerwick, "Contraction mappings in b-metric spaces," Acta Mathematica et Informatica Universitatis Ostraviensis, vol. 1, pp. 5-11, 1993.

[3] A. Azam, B. Fisher, and M. Khan, "Common fixed point theorems in complex valued metric spaces," Numerical Functional Analysis and Optimization, vol. 32, no. 3, pp. 243-253, 2011.

[4] K. P. R. Rao, P. R. Swamy, and J. R. Prasad, "A common fixed point theorem in complex valued b-metric spaces," Bulletin of Mathematics and Statistics Research, vol. 1, no. 1, 2013.

[5] P. Dhivya and M. Marudai, "Common fixed point theorems for mappings satisfying a contractive condition of rational expression on a ordered complex partial metric space," Cogent Mathematics, vol. 4, Article ID 1389622, 2017.

[6] M. Gunaseelan, "Generalized fixed point theorems on complex partial b-metric space," International Journal of Research and Analytical Reviews, vol. 6, no. 2, 2019.

[7] Deepmala and H. K. Pathak, "A study on some problems on existence of solutions for nonlinear functional-integral equations," Acta Mathematica Scientia, vol. 33, no. 5, pp. 1305-1313, 2013.

[8] Deepmala, "A Study on fixed point theorems for nonlinear contractions and its applications," Ph.D. thesis, Pt. Ravishankar Shukla University, Raipur 492 010, Chhatisgarh, India, 2014.
[9] H. K. Pathak and Deepmala, "Common fixed point theorems for," Journal of Computational and Applied Mathematics, vol. 239, pp. 103-113, 2013.

[10] A. Latif, T. Nazir, and M. Abbas, "Stability of fixed points in generalized metric spaces," Journal of Nonlinear Var. Analysis, vol. 2, pp. 287-294, 2018.

[11] M. Abbas, I. Beg, and B. T. Leyew, "Common fixed points of $(\mathrm{R}, \alpha)$-generalized rational multivalued contractions in R-complete b-metric spaces," Communications in Optimization Theory, vol. 2019, 14 pages, 2019.

[12] F. Gu and W. Shatanawi, "Some new results on common coupled fixed points of two hybrid pairs of mappings in partial metric spaces," Journal of Nonlinear Functional Analysis 2000 Mathematics Subject Classification. 47H10, vol. 2019, Article ID 13, 2019.

[13] T. Gnana Bhaskar and V. Lakshmikantham, "Fixed point theorems in partially ordered metric spaces and applications," Nonlinear Analysis: Theory, Methods and Applications, vol. 65, no. 7, pp. 1379-1393, 2006.

[14] M. Gunaseelan and L. N. Mishra, "Coupled fixed point theorems on complex partial metric space using different type of contractive conditions," Scientific Publications of the State University of Novi Pazar Series A: Applied Mathematics, Informatics and Mechanics, vol. 11, no. 2, pp. 117-123, 2019. 